\title{
Maternal mortality and derivations from the WHO near-miss tool: An institutional experience over a decade in Southern India
}

\author{
Ajay Halder ${ }^{1}$, Ruby Jose ${ }^{2}$, Reeta Vijayselvi ${ }^{2}$ \\ 'Department of Obstetrics and Gynecology, All India Institute of Medical Sciences, Bhopal, India \\ ${ }^{2}$ Department of Obstetrics and Gynecology, Christian Medical College, Vellore, India
}

\section{Abstract}

Objective: Preceding the use of World Health Organization (WHO) near-miss approach in our institute for the surveillance of Severe Maternal Outcome (SMO), we pilot-tested the tool on maternal death cases that took place over the last 10 years in order to establish its feasibility and usefulness at the institutional level.

Material and Methods: This was a retrospective review of maternal deaths in Christian Medical College Vellore, India, over a decade. Cases were recorded and analyzed using the WHO near-miss tool. The International Classification of Diseases, $10^{\text {th }}$ Revision was used to define and classify maternal mortality.

Results: There were 98,139 total births and 212 recorded maternal deaths. Direct causes of mortality constituted $46.96 \%$ of total maternal deaths, indirect causes constituted $51.40 \%$, and unknown cases constituted $1.9 \%$. Nonobstetrical cause (48.11\%) is the single largest group. Infections (19.8\%) other than puerperal sepsis remain an important group, with pulmonary tuberculosis, scrub typhus, and malaria being the leading ones. According to the WHO near-miss criteria, cardiovascular and respiratory dysfunctions are the most frequent organ dysfunctions. Incidence of coagulation dysfunction is seen highest in obstetrical hemorrhage (64\%). All women who died had at least one organ dysfunction; $90.54 \%$ mothers had two- and $38.52 \%$ had four- or more organ involvement.

Conclusion: The screening questions of the WHO near-miss tool are particularly instrumental in obtaining a comprehensive assessment of the problem beyond the International Classification of Diseases-Maternal Mortality and establish the need for laboratory-based identification of organ dysfunctions and prompt availability of critical care facilities. The process indicators, on the other hand, inquire about the basic interventions that are more or less widely practiced and therefore give no added information at the institutional level.

(J Turk Ger Gynecol Assoc 2014; 15: 222-7)

Key words: Maternal mortality, who near-miss tool, organ dysfunction

Received: 19 April, 2014

Accepted: 28 September, 2014

\section{Introduction}

A state's health status can largely be assessed by the incidence of its maternal mortality. The Millennium Development Goals (MDG) of the United Nations (1) had set the goal of $109 / 100,000$ live births by 2015 . In this regard, India and many of its most populous states have performed fairly under national government initiatives, like the National Rural Health Mission (NRHM), Janani Shishu Suraksha Yojana (conditional cash transfer scheme), and Accredited Social Health Activists schemes (2). But, after the initial success, India may have to wait until 2023-2024 to attain the targeted maternal mortality ratio (MMR) if it fails to continue the linear declining tread achieved in 1997-2009 (3).

The study of "near-miss" and maternal death cases can provide useful insights into processes that can lead to maternal adverse outcomes (4). In 2007, the WHO established a techni- cal working group to develop a standard definition and uniform identification criteria for maternal near-miss cases. The near-miss identification criteria thus developed target cases presenting with features of severe organ dysfunctions (5). It has been shown to yield useful and reliable data that can be used to improve the quality of care and monitor maternal health care interventions.

Preceding the use of the WHO near-miss approach in our institute for the surveillance of severe maternal outcomes, we pilot-tested the tool over the maternal death cases that took place over the last 10 years in order to establish its feasibility and usefulness at the institutional level.

\section{Material and Methods}

This is a retrospective study based on data from the labor ward, intensive care unit, discharge summaries, and admis- 
sion records maintained by the medical records unit of Christian Medical College (CMC), Vellore, India. Situated in the southern India, CMC is a tertiary referral center and one of the oldest medical college hospitals in India. The hospital receives referrals mainly from the southern parts of the country. All mortality statistics in the concerned departments were compiled into an Epi Info database using the WHO near-miss tool (6) as the format. All maternal deaths following admission to the CMC from January 1, 2003 to December 31, 2012 were reviewed and analyzed. The CMC's maternal mortality audit team, in collaboration with the medical records unit, identified the maternal deaths. The case records with deficient information were used to calculate the MMR and the underlying cause of death only. Identification and classification of Maternal Death- Direct and Indirect, along with further subdivisions (nine subgroups), were done according to WHO application of International Classification of Disease (ICD) to deaths during pregnancy, childbirth, and the puerperium: ICD-Maternal Mortality (ICD-MM) which is based upon the 10th revision of the ICD (ICD-10) (7).

\section{Data analysis}

Maternal mortality data were analyzed using Excel (Microsoft; Washington, USA) spreadsheet. Proportions and maternal mortality rates per 100,000 live births were calculated. Where more than one cause for death was recorded, only the first or the underlying cause (ICD-MM) of death was considered. Other secondary or tertiary causes of the obstetric death were noted if they satisfied the WHO near-miss criteria (6), which are further classified into a) potentially life-threatening conditions, b) critical interventions, and c) organ dysfunction criteria. Readers are advised to read through the sample data collection form in the WHO document (8) for maximum benefit from the discussion below.

Institutional ethical committee permission was sought and obtained for the study. The study was not funded. The authors declare that there is no conflict of interest.

\section{Result}

There were 98,139 total births and 95,384 live births between 2003 and 2012. During this period, there were 28,788 cesarean deliveries and an average perinatal mortality rate of 35,391 per 1000 live births. There were 212 recorded maternal deaths during this period. The yearly maternal mortality ratio per 100,000 live births per year is shown in Table 1. The mean age at death was 24 years (standard deviation (SD) 4.4), mean parity was 1 (SD 0.97), and the mean period of gestation at delivery or death was 24 weeks (SD 8.15). The distribution of age, parity, period of gestation at death or birth, end of pregnancy mode, fetal outcome, and comorbidities is shown in Tables 2 and 3. Perinatal mortality was seen in $69 \%$ of cases.

The direct causes of mortality constituted $46.96 \%$ (99), the indirect causes constituted $51.40 \%$ (109), and unknown cases constituted 1.9\% (4) (Table 4). It is interesting to note that the percentage distribution of the causes of maternal deaths did not change significantly over the decade, as depicted in Figure 1, where the causes have been shown against the three 40-month
Table 1. Maternal mortality ratio from 2003 to 2012

\begin{tabular}{|l|c|c|c|}
\hline Year & $\begin{array}{c}\text { Total live } \\
\text { births }\end{array}$ & $\begin{array}{c}\text { Number of } \\
\text { maternal deaths }\end{array}$ & $\begin{array}{c}\text { Maternal mortality } \\
\text { ratio (per 100,000 } \\
\text { live births) }\end{array}$ \\
\hline 2003 & 7758 & 29 & 374 \\
\hline 2004 & 7938 & 17 & 214 \\
\hline 2005 & 7625 & 18 & 236 \\
\hline 2006 & 7753 & 25 & 322 \\
\hline 2007 & 8629 & 18 & 208 \\
\hline 2008 & 9316 & 15 & 161 \\
\hline 2009 & 10,068 & 27 & 278 \\
\hline 2010 & 11,115 & 22 & 198 \\
\hline 2011 & 12,099 & 18 & 148 \\
\hline 2012 & 13,084 & 26 & 198 \\
\hline Total & 95,385 & 215 & 222 \\
\hline
\end{tabular}

Table 2. Maternal age, parity, and gestational age at delivery/death

\begin{tabular}{|l|c|}
\hline Maternal age & $149(100 \%)$ \\
\hline $15-19$ & $12(8 \%)$ \\
\hline $20-24$ & $67(45 \%)$ \\
\hline $25-29$ & $46(31 \%)$ \\
\hline $30-34$ & $19(13 \%)$ \\
\hline $35-39$ & $5(3 \%)$ \\
\hline Parity & $135(100 \%)$ \\
\hline 0 & $80(59 \%)$ \\
\hline 1 & $33(24 \%)$ \\
\hline 2 or more & $22(16 \%)$ \\
\hline Gestational age at delivery or death & $149(100 \%)$ \\
\hline 12 weeks or less & $9(6 \%)$ \\
\hline $13-28$ weeks & $19(13 \%)$ \\
\hline $29-34$ weeks & $28(18 \%)$ \\
\hline $35-41$ weeks & $94(68 \%)$ \\
\hline
\end{tabular}

time periods. The killer trio hemorrhage, puerperal sepsis, and hypertension maintained their position as the leaders of death due to direct causes. These three together caused $37.1 \%$ of maternal deaths in our hospital over the last decade. Deaths related to abortions have shown a decline, and no such case was seen in the last 40-month period.

ICD-MM group VI "Non-Obstetrical cause" [48.11\% (102/212)] is the single largest group, composed of numerous common and uncommon diseases. Rheumatic heart disease and fulminant liver disease were the most important medical conditions resulting in maternal death in this group. Infections [19.8\% (42/212)] other than puerperal sepsis remain an important group, with pulmonary tuberculosis, scrub typhus, and malaria 
Table 3. Mode of delivery, fetal outcome, and associated comorbidities

\begin{tabular}{|l|c|}
\hline Final mode of delivery & $147(100 \%)$ \\
\hline Vaginal & $70(48 \%)$ \\
\hline Cesarean section & $47(32 \%)$ \\
\hline Abortion & $10(7 \%)$ \\
\hline Laparotomy for perforation & $3(2 \%)$ \\
\hline Discharged or died pregnant & $17(12 \%)$ \\
\hline Fetal outcome & $143(100 \%)$ \\
\hline Live birth & $43(30 \%)$ \\
\hline Stillborn & $84(58 \%)$ \\
\hline Early neonatal death & $16(11 \%)$ \\
\hline Associated comorbidities & $145(100 \%)$ \\
\hline Anemia & $77(53 \%)$ \\
\hline HIV positive & $4(2.7 \%)$ \\
\hline Prolonged labor & $5(3.4 \%)$ \\
\hline Previous LSCS & $8(5.5 \%)$ \\
\hline $\begin{array}{l}\text { LSCS: lower section caesarean section; HIV: human immunodeficiency } \\
\text { virus }\end{array}$ & \\
\hline
\end{tabular}

being the leading ones. There are clustered cases of maternal deaths due to H1N1 during an outbreak. Heat stroke caused 6 maternal deaths during the 10-year period. The higher percentage of maternal deaths due to medical conditions (Group 7 ICD-MM O98) was probably due to the presence of a large proportion of women with medical conditions in a tertiary referral hospital.

According to the WHO near-miss criteria, the organ dysfunctions encountered before the maternal death are shown in Figure 2. Cardiovascular and respiratory dysfunctions were the most frequently seen organ dysfunctions, either being an underlying cause or a later sequela. Among the six main primary causes, cardiovascular dysfunction was present in 53.73\% (108/201) of cases and respiratory dysfunction was present in $60.19 \%$ (121/201). Coagulation dysfunction in the form of failure to form clots, massive transfusion of blood or red cells ( $\geq 5$ units), and severe acute thrombocytopenia $(<50,000$ platelets $/ \mathrm{ml})$ was seen in a significant percentage (36.31\%) of mothers who subsequently died, with the highest percentage in the mothers of group III obstetrical hemorrhage (64\%). Uterine dysfunction, defined as hemorrhage or infection leading to hysterectomy, was seen in $32 \%$ of cases in group III obstetrical hemorrhage. Hysterectomy was also done in $7.15 \%, 3.8 \%$, and $3.9 \%$ of cases in group I pregnancy with abortive outcomes, group II hypertensive disorders, and group VII non-obstetric complications,

Table 4. Causes of maternal death according to International Classification of Diseases $10^{\text {th }}$ Revision (ICD-10 \& ICD-MM)

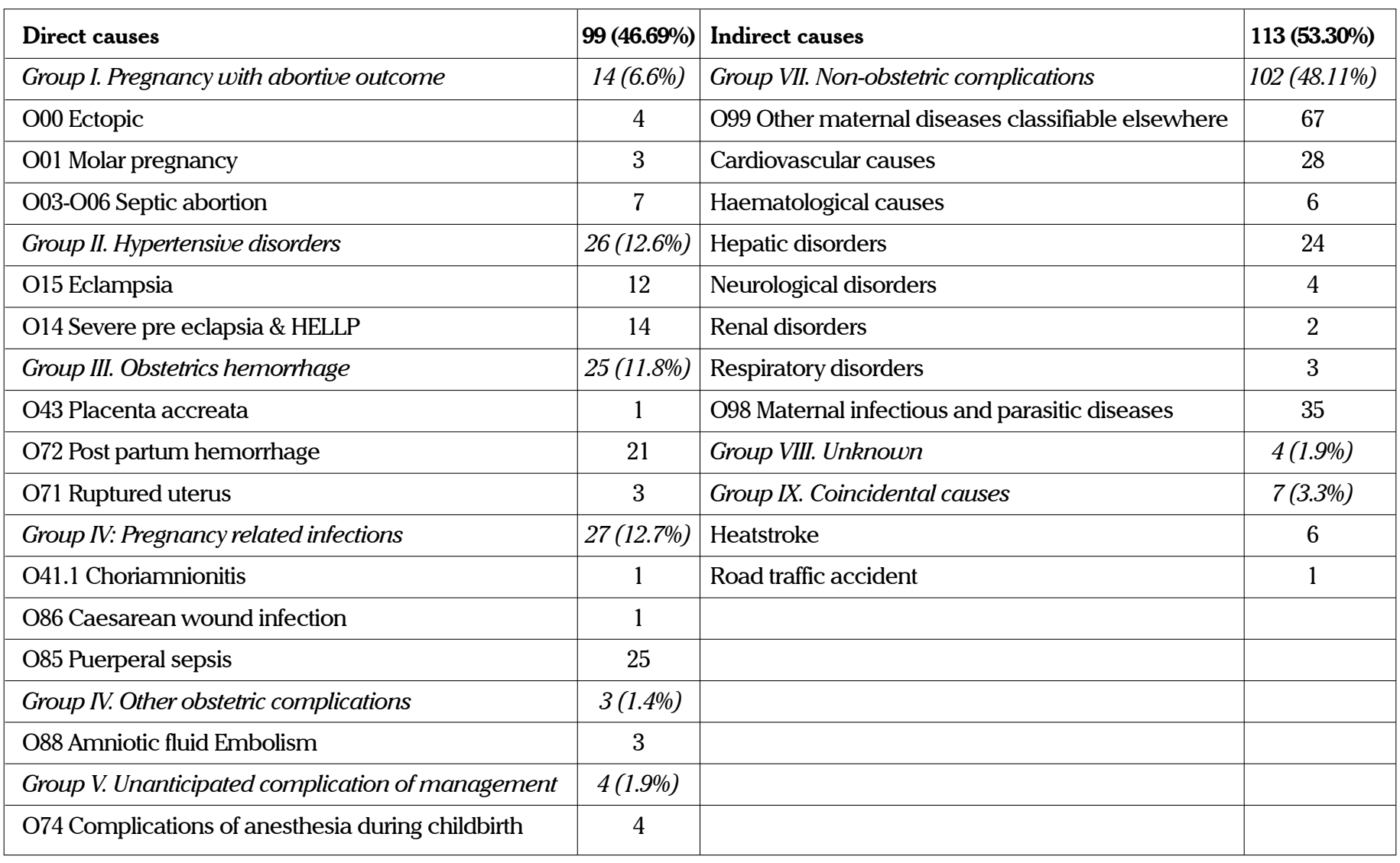


respectively. Neurological dysfunction was exceptionally high in group II hypertensive disorder (46.2\%) and group IV pregnancyrelated infections (25.9\%) compared to other major ICD-MM groups $(8.5 \%$ to $16 \%)$. All women who died had at least one organ dysfunction; $90.54 \%$ mothers had two- and $38.52 \%$ had four- or more organ involvement (Figure 3). WHO screening criteria other than organ dysfunction was not uniformly present in all maternal deaths. The life-threatening conditions and critical interventions, if used alone, would have missed 24\% (36/150) and $8.6 \%$ (13/150) of cases, respectively (Figure 4).

Seventy-nine percent (26/33) of the deaths that took place within 12 hours of admission were women referred from outside. The average time since delivery to death (days) showed a declining trend, whereas the average duration of hospital stay showed a significant increasing trend over the decade (Figure 5).

\section{Discussion}

To counter the stagnation in the decline of maternal mortality in many growing economies, like India, a pre-emptive approach to identify and treat maternal near-miss events seems prudent. In 2009, the WHO working group on maternal

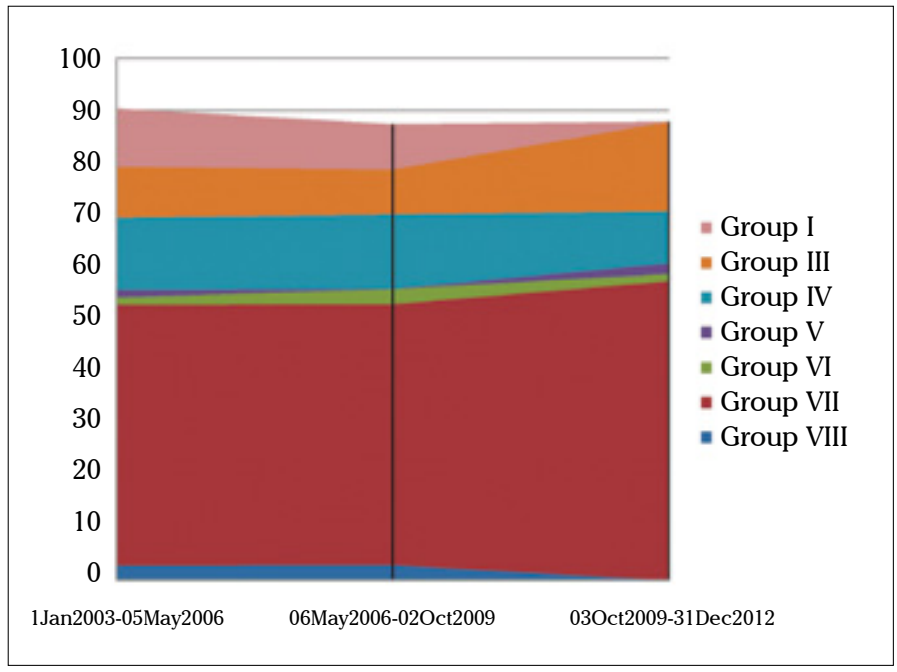

Figure 1. Trends in maternal mortality cases (ICD-MM groups) morbidity and mortality classifications (6) put forth the WHO near-miss criteria containing 25 severity markers, primarily laboratory-based, that were shown to be independently associated with poor maternal outcome. Cecatti et al. (9), in a prospective study of 673 cases of severe maternal morbidity, tested the performance of the WHO criteria against the SOFA score $(10,11)$, the gold standard for organ dysfunction identification in intensive care settings, and found it to be $100 \%$ sensitive and $70.4 \%$ specific for predicting maternal death cases. Sauza et al. (12), in their prospective study across 27 referral centers in a Latin American country, used a binary logistic regression model to describe the association between

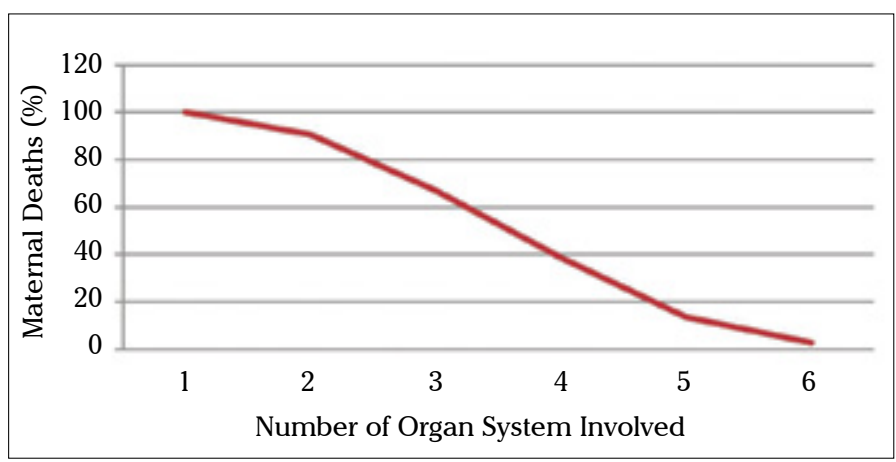

Figure 3. Organ dysfunction(s) in maternal mortality (WHO Criteria)

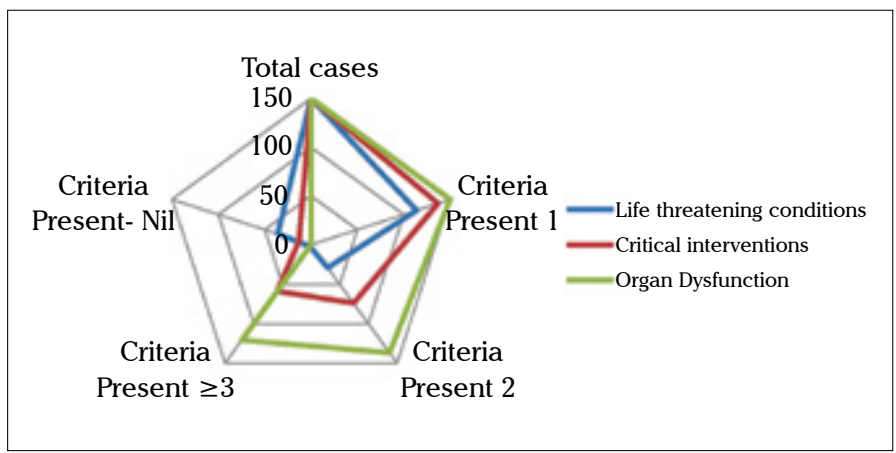

Figure 4. WHO screening criteria in maternal mortality (Ability of different groups of screening criteria to identify mortality cases)

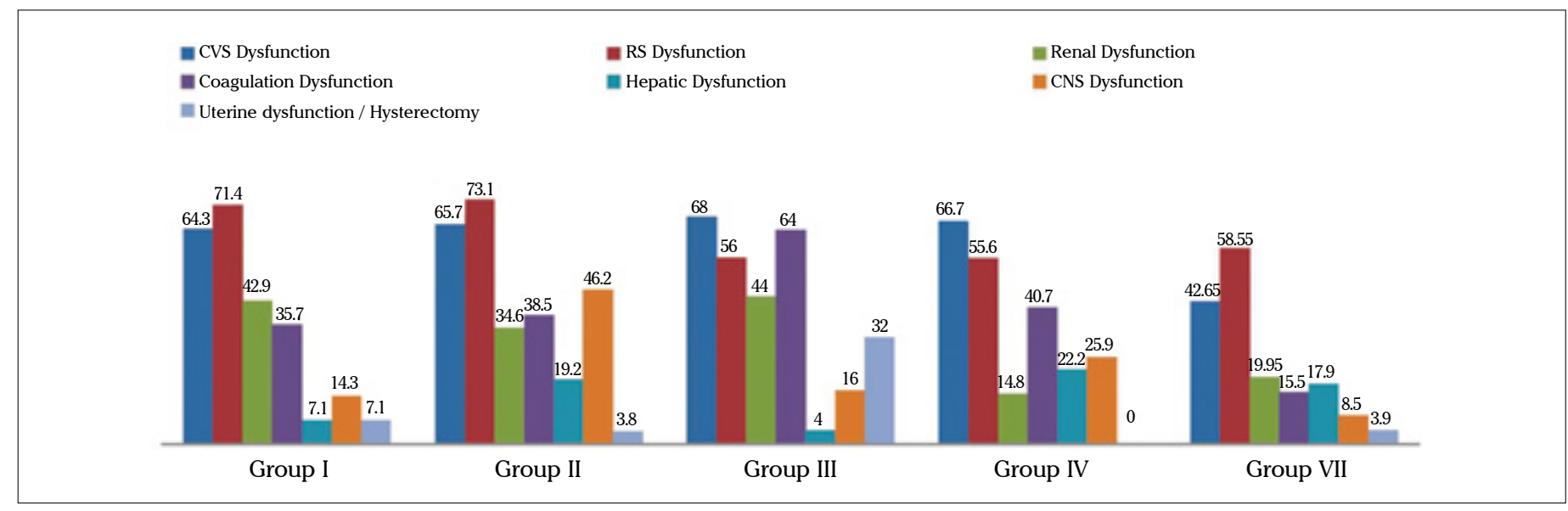

Figure 2. Organ dysfunction (\%) in major ICD-MM groups 


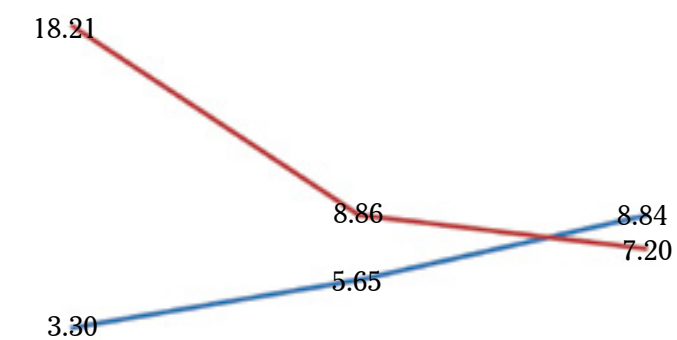

1Jan2003-05May2006 06May2006-02Oct2009 03Oct2009-31Dec2012

— Hospital Stay _ Time since Delivery

Figure 5. Hospital stay vs time since delivery (Days)

severe maternal outcome and WHO near-miss criteria. With a positive likelihood ratio of 106.8 (95\% CI 99.56-114.6), the WHO near-miss criteria had a high association with maternal deaths. The presence of at least one organ dysfunction in every maternal mortality case in the present study seconds the findings of Sauza et al. (12). A maternal severity index model was also proposed by Sauza et al. (12) that predicted the probability of maternal death with complications of pregnancy. The WHO Multicountry Survey (WHOMCS) (13) on maternal and newborn health was conducted across 29 countries in Asia, Africa, and Latin America among 357 centers and showed that older, less educated, and higherparity mothers with cesarean deliveries were more likely to have a severe maternal outcome (SMO). Perinatal outcome was dismal in SMO cases, with a 15 times higher perinatal mortality rate and a proportionate increase in preterm labor and neonatal intensive care unit admissions. Postpartum hemorrhage and hypertensive disorders of pregnancy were the most common obstetric complications. The incidence of sepsis and systemic infections was higher in comparison to puerperal endometritis, similar to the observations of the present study. Cardiovascular, respiratory, and coagulation dysfunctions were the most common organ dysfunctions, as also seen in the present study. Our study could further show that the spectrum of organ dysfunction across all major ICDMM groups was similar, with very few exceptions, like higher coagulation disorder in the obstetric hemorrhage group and neurologic dysfunction in the hypertensive group. This dilutes the importance of classifying maternal deaths by the underlying cause and establishes the need for laboratory-based identification of organ dysfunction and prompt availability of critical care facilities. In spite of the high coverage of the indicated essential interventions (process indicators) across the health facilities, the WHOMCS showed unequal performance regarding maternal mortality. Furthermore, in the instances where the indicated essential interventions were missed in SMO cases (missed opportunities), the risk of death was not higher. This questions the relevance of the indicated essential interventions in reducing maternal mortality further beyond a limit. The WHOMCS included women in early puerperium up to 7 days postpartum and may have missed late puerperal cases of SMO, which by definition is up to 42 days.
The present study was limited by its retrospective design and the incomplete information in the medical records. Since this study was conducted with only mortality cases, the extrapolation of the findings to all pregnant women remains hypothetical. Nevertheless, in light of the previous works, this study gathers further support in favor of the use of the WHO maternal near-miss approach.

The WHO screening questions, composed of potentially lifethreatening conditions, critical interventions, and organ dysfunction criteria, are particularly instrumental in obtaining a comprehensive assessment of the problem beyond the ICD-MM. The use of criteria on potentially life-threatening conditions alone, however, does not add information above what is already provided by the section on underlying cause of death/near-miss. The process indicators, especially the use of interventions, on the other hand, inquires about basic interventions, which are more or less widely practiced and therefore gives no added information.

The approach to improving maternal health is ideally through defining, quantifying, and taking measures to reduce severe maternal outcomes, which include both maternal near-miss and death. Provided that basic antenatal care and emergency obstetric care is available to the majority, further success will follow only a more aggressive approach in averting maternal mortality by identifying maternal near-miss and providing advanced life support to mothers with severe organ dysfunctions.

Ethics Committee Approval: Ethics committee approval was received for this study.

Informed Consent: N/A.

Peer-review: Externally peer-reviewed.

Author contributions: Concept - A.H., R.J.; Design - A.H., R.J.; Supervision - A.H., R.J., R.V.; Resource - A.H., R.V.; Materials - A.H., R.V.; Data Collection\&/or Processing - A.H., R.J., R.V.; Analysis\&/or Interpretation A.H., R.J., R.V.; Literature Search - A.H., R.V.; Writing - A.H., R.J.; Critical Reviews - A.H., R.J.

Acknowledgements: We thank Dr Abhijeet Pakhare for his significant contribution in data analysis and interpretation and Mrs Alice Augustine for her significant contribution in data collection.

Conflict of Interest: No conflict of interest was declared by the authors.

Financial Disclosure: The authors declared that this study has received no financial support.

\section{References}

1. United Nations Development Fund. Millennium development goals. Available from: http://www.undp.org/mdg/basics.shtml.

2. Shiffman J, Ved R. The state of political priority for safe motherhood in India. BJOG 2007; 114: 785-90. [CrossRef]

3. Reddy H, Pradhan MR, Ghosh R, Khan A G. India's progress towards the Millennium Development Goals 4 and 5 on infant and maternal mortality. WHOSEAJPH 2012; 1: 279-89.

4. Say L, Pattinson RC, Gülmezoglu AM. WHO systematic review of maternal morbidity and mortality: the prevalence of severe acute maternal morbidity (nearmiss). Reprod Health 2004; 1: 3. [CrossRef]

5. Pattinson R, Say L, Souza JP, Broek N, Rooney C. WHO maternal death and nearmiss classifications. Bull World Health Organ 2009; 87: 734. [CrossRef] 
6. Say L, Souza JP, Pattinson RC, WHO working group on Maternal Mortality and Morbidity classifications. Maternal near miss - towards a standard tool for monitoring quality of maternal health care. Best Pract Res Clin Obstet Gynaecol 2009; 23: 287-96. [CrossRef]

7. The WHO Application of ICD-10 to deaths during pregnancy, childbirth and the puerperium: ICD-MM. Available from: http://www.who. int/reproductivehealth/publications/monitoring/9789241548458/en/

8. Evaluating the quality of care for severe pregnancy complications: the WHO near-miss approach for maternal health. Available from: http://www.who.int/reproductivehealth/topics/maternal_perinatal/ nmconcept/en/

9. Cecatti JG, Souza JP, Oliveira Neto AF, Parpinelli MA, Sousa MH, Say L, Pattinson RC. Pre-validation of the WHO organ dysfunction based criteria for identification of maternal near miss. Reprod Health 2011; 8: 22. [CrossRef]

10. Vincent J-L, De Mendonça A, Cantraine F, Moreno R, Takala J, Suter $\mathrm{PM}$, et al. Use of the SOFA score to asses the incidence of organ dysfunction/failure in intensive care units: Results of a multicentric, prospective study. Working group on "sepsis-related problems" of the European Society of Intensive Care Medicine. Crit Care Med 1998, 26: 1783-800. [CrossRef]

11. Oliveira Neto AF, Parpinelli MA, Cecatti JG, Souza JP, Sousa MH. Sequential organ failure assessment (SOFA) score for evaluating organ failure and outcome in severe maternal morbidity in obstetric intensive care. ScientificWorldJournal 2012; 2012: 172145. [CrossRef]

12. Souza JP, Cecatti JG, Haddad SM, Parpinelli MA, Costa ML, Katz L, et al. The WHO maternal near-miss approach and the maternal severity index model (MSI): tools for assessing the management of severe maternal morbidity. PLoS One 2012; 7: e44129. [CrossRef]

13. Souza JP, Gülmezoglu AM, Vogel J, Carroli G, Lumbiganon P, Qureshi Z, et al. Moving beyond essential interventions for reduction of maternal mortality (the WHO Multicountry Survey on Maternal and Newborn Health): a cross-sectional study. Lancet 2013; 381: 1747-55. [CrossRef] 